\title{
Screening for Opioid Use Disorder in the Largest Jail in Arkansas: A Brief Report
}

\author{
Nickolas Zaller, PhD', Kristin Donadeo, BA², James Coffey, MA', \\ Melissa Zielinski, $\mathrm{PhD}^{1,3}$, and Lauren Brinkley-Rubinstein, $\mathrm{PhD}^{4}$
}

\begin{abstract}
Individuals who have experienced incarceration have an increased risk of both fatal and nonfatal overdose. Given the increases in illicit opioid use in Arkansas and across the South, many individuals with opioid use disorder (OUD) are likely to encounter the criminal justice system, particularly county jails. However, there are currently no published data on OUD among entrants into county jails in the South. This study presents data from an OUD screening project initiated by the Pulaski County Regional Detention Facility, the largest county jail in the state of Arkansas. As part of the regular intake process, a jail staff person administered the Rapid Opioid Dependence Screen. De-identified data were provided to researchers at the University of Arkansas for Medical Sciences. The data clearly show an increased prevalence of OUD, justifying the need for evidence-based substance use programs such as MAT in jail settings in the South.
\end{abstract}

\section{Keywords}

opioid use disorder, jail, intake screening, correctional health care

\section{Background}

The United States is in the midst of an epidemic of opioid use and subsequent opioid overdose. In 2015, over 12.5 million people were affected by the opioid epidemic, and approximately 33,000 people died of an overdose involving opioids (Centers for Disease Control and Prevention [CDC], 2018). Individuals who have experienced incarceration have an increased risk of both fatal and nonfatal overdose (Binswanger et al., 2007). The immediate postrelease period is a time of extreme risk because many people return to substance use after imposed abstinence during incarceration and lack of stability and access to resources in the community (Binswanger et al., 2007; Degenhardt et al., 2014).

\footnotetext{
' College of Public Health, University of Arkansas for Medical Sciences, Little Rock, AR, USA

${ }^{2}$ University of Arkansas, Little Rock, AR, USA

${ }^{3}$ Psychiatric Research Institute, University of Arkansas for Medical Sciences, Little Rock, AR, USA

${ }^{4}$ School of Medicine, University of North Carolina, Chapel Hill, NC, USA
}

\section{Corresponding Author:}

Lauren Brinkley-Rubinstein, PhD, I2I Main St., Providence, RI 02906, USA.

Email: lauren_brinkley@med.unc.edu 
To address the risk of opioid overdose during community reentry, correctional facilities (mostly prisons) in some locations across the United States have started to implement programs that provide access to medication-assisted treatment (MAT; National Governors Association, 2017). However, nearly all of these programs are in geographic regions outside of the South, and few are present in county jails, which hold individuals for relatively short periods (days to months) prior to sentencing. For example, no jail or prison in the state of Arkansas provides MAT; individuals on MAT at the time of incarceration are detoxed upon entrance to correctional facilities. The Southern region has both the highest rates of incarceration and escalating rates of opioid use and overdose (National Institute on Drug Abuse, 2019; Nellis, 2016). In 2015, the South recorded 5,374 overdose-related deaths, the highest number of any region in the United States (CDC, 2018). Arkansas, the focus of this article, has the second highest rate of opioid prescriptions in the United States (114.6 prescriptions per 100 people; CDC, 2017).

Given the increases in illicit opioid use in Arkansas and across the South, many individuals with opioid use disorder (OUD) are likely to come into contact with the criminal justice system, particularly county jails (Brinkley-Rubinstein, Zaller, et al., 2018). However, there are currently no published data on OUD among entrants into county jails in the South. Thus, we present data from an OUD screening project initiated by the Pulaski County Regional Detention Facility (PCRDF), the largest county jail in the state of Arkansas, with technical assistance from the University of Arkansas for Medical Sciences. The PCRDF is working toward developing a MAT program for OUD jail detainees, and this screening project was conducted in order to ascertain baseline estimates of OUD among individuals entering the jail.

\section{Method}

Data reported here are from screenings conducted during March and April 2017. During this time, the PCRDF screened individuals for OUD upon intake. As part of the regular intake process, a jail staff person administered the Rapid Opioid Dependence Screen (RODS). The RODS is an 8-item measure of opioid dependence, based on diagnostic criteria outlined in the DSM-IV. Individuals are categorized as opioid dependent if they have at least three affirmative responses to Items 2 to 8 . The RODS tool was designed to be rapidly administered ( $\sim 2 \mathrm{~min}$ ) and has been used in correctional settings (Wickersham, Azar, Cannon, Altice, \& Springer, 2015). In addition, the jail collected basic demographic data including race/ethnicity, age, and gender for reporting purposes. The jail shared de-identified data with the authors for analysis. We conducted descriptive data analysis by tabulating the frequency of demographic characteristics, use of specific opioids, and opioid dependence among the sample. SPSS Statistics Version 24 (IBM) software was used for statistical analysis of these data. The University of Arkansas for Medical Sciences institutional review board approved this study.

\section{Results}

During the study period, the RODS was administered to 978 individuals at jail intake. Data were not provided regarding the total number of jail entrants during this time period. However, in 2016, there were approximately 2,000 individuals admitted per month. Thus, we estimate that staff administered the RODS to approximately 17\% of individuals admitted to the jail between March and April 2017. The median age was 32 years old, and the majority of the sample was male $(70.9 \%)$ and Black or African American (59.2\%). A total of 92, or 9.3\% of the total sample, screened positive for opioid dependence. The most commonly reported substances were heroin, oxycodone, and other opioids, not specified. Among individuals reporting use of methadone or buprenorphine, we do not have data to indicate whether these opioids were used due to participation in a MAT program at the time of arrest. 
Table I. Demographic Characteristics of Detainees at the Pulaski County Jail.

\begin{tabular}{lccc}
\hline & $\begin{array}{c}\text { Opioid-Dependent } \\
\text { Respondents }(N=92)\end{array}$ & $\begin{array}{c}\text { Nonopioid-Dependent } \\
\text { Respondents }(N=886)\end{array}$ & $p^{\mathrm{a}}$ \\
\hline $\begin{array}{l}\text { Median age (range) } \\
\text { Gender }(\%)\end{array}$ & $33(18-74)$ & .22 \\
$\quad$ Male & $32(18-54)$ & $640(92.7)$ & .004 \\
$\quad$ Female & $50(7.3)$ & $196(86.3)$ & \\
Race $^{\text {b }} \%$ & $31(13.7)$ & $599(97.2)$ & \\
$\quad$ Non-White & & $261(78.6)$ & \\
$\quad$ White & $17(2.8)$ & $25(86.2)$ & .001 \\
Ethnicity $(\%)$ & $71(21.4)$ & $831(91.0)$ & \\
$\quad$ Hispanic or Latino & $4(13.8)$ & & \\
Not Hispanic or Latino & $82(9.0)$ & & \\
\hline
\end{tabular}

Note. ${ }^{a}$ Missing data.

Gender: $6 \mathrm{I}$ respondents did not identify as male or female or refused to answer the question-nonbinary $(n=I)$, prefer not to say $(n=58)$, missing $(n=30)$.

Race: 30 respondents-Non-White includes African American $(n=12)$ and American Indian/Alaska Native $(n=2)$, Asian $(n$ $=1$ ), Native Hawaiian or other Pacific Islander $(n=I)$, more than one race $(n=I)$, and unknown/not reported $(n=35)$. Ethnicity: 36 respondents.

${ }^{\mathrm{b}}$ To assess the association between age and opioid dependence, we conducted a two-sample $t$ test with equal variances. For all other categorical variables, we conducted a Fischer's exact $\chi^{2}$ test.

Table 1 depicts differences in demographic characteristics between those who screened opioid dependent and those who did not. Overall, there were statistically significant differences for both gender and race. Both women and Whites had a greater likelihood of screening positive for opioid dependence relative to men and other races, respectively. Importantly, Whites had a more than sevenfold higher proportion of opioid dependence relative to non-Whites $(21.4 \%$ vs. $2.8 \%, p<.001)$. Additionally, a much higher proportion of women were opioid dependent $(13.7 \%$ vs. $7.3 \%$, $p=.004)$.

\section{Discussion}

To our knowledge, this is the first reported OUD prevalence estimate from a county jail in the South. Given that many substance users come into contact with county jails (Bronson, Stroop, Zimmer, \& Berzofsky, 2017), these institutions are in an important position to provide screening and treatment for opioid dependence. While estimates of OUD may be difficult to obtain at the county level, recent data from the National Survey on Drug Use and Health (NHSDU) administered by the Substance Abuse and Mental Health Services Administration suggest that Arkansas has an illicit substance use prevalence of $1.7 \%$ (Nellis, 2016). Thus, the OUD prevalence of $9.3 \%$ in our sample of jail detainees is nearly 5.5 times greater than the state prevalence of substance dependence for all illicit substances combined. However, our jail population had a greater proportion of both African Americans and men, respectively, relative to the NHSDU Arkansas state substance use prevalence estimates.

While our findings are not surprising, understanding the magnitude of OUD among jail detainees is a crucial first step toward developing critical intervention strategies, such as MAT within the jail setting and/or targeting for overdose education and naloxone provision prior to release. Our results also demonstrate that White (non-Hispanic) women were more likely to screen positive for OUD, which is inconsistent with state-level data for treatment admission in that nearly three quarters of OUD treatment admissions in 2016 were among White (non-Hispanic) men (Arkansas Department of Health and Human Services, P. Brannan, personal communication, 2016). Of importance, routine 
and universal substance use screening of detainees will be an important next step as jails further contemplate implementation of treatment programming. Our data suggest that OUD screening was not necessarily done in a uniform manner and likely missed significant numbers of detainees at intake. Furthermore, jail administrators will need to identify optimal candidates for MAT, for example, those who will be incarcerated long enough to go through MAT induction. Finally, partnerships between the jail and regional MAT providers will need to be strengthened to ensure appropriate linkage to MAT upon release from jail. Currently, there is a scarcity of MAT providers in both Pulaski County and in the rest of Arkansas, which means that investment in postrelease linkage to community treatment should be considered.

\section{Limitations}

Our estimates of OUD among jails detainees may underrepresent the true prevalence since some individuals may have had reservations about reporting substance use to jail personnel during the intake process. Furthermore, jail staff screened only approximately one fifth of the total number of individuals admitted to the jail during the 2-month study period. This may be due to the high turnover in jail populations and the fact that many detainees are incarcerated for very brief periods, that is, less than $24 \mathrm{hr}$. Additionally, the screening was done as part of a chaotic jail intake process, and jail staff may not have been able to offer the screening at all times to all individuals. Therefore, we recognize that our sample may not be representative of the total Pulaski County jail population. Finally, we did not collect information about types of arrest charges among detainees, so we do not have data to corroborate self-reported substance use. More research is needed to determine whether these results are similar to other jail settings in the South.

\section{Conclusion}

One of the major priorities of the U.S. Department of Health and Human Services is to combat the epidemic of OUD and improve access to treatment and recovery services (U.S. Department of Health and Human Services, Office of the Surgeon General, 2016). People with recent incarceration are at increased risk of overdose, and provision of MAT during incarceration is an effective way to stem risk during community reentry (Brinkley-Rubinstein, McKenzie, et al., 2018; Marsch, 1998; Mattick, Kimber, Breen, \& Davoli, 2008; Rich et al., 2015). However, in the South, where incarceration rates and opioid use are among the highest in the country, little is known about baseline rates of OUD in correctional settings. Our data clearly show that there is an increased prevalence of OUD justifying the need for evidence-based substance use programs such as MAT in these settings.

\section{Acknowledgments}

We would like to thank the Pulaski County Regional Detention Facility for their assistance with this project. In particular, we would like to acknowledge Lt. Ron Routh (Pulaski County Regional Detention Facility), Dr. Jeremy Thompson (TurnKey, Inc.), and Mr. James Pratt (formerly of TurnKey, Inc.), who were instrumental in conducting the study.

\section{Declaration of Conflicting Interests}

The authors disclosed no conflicts of interest with respect to the research, authorship, or publication of this article. For information about $J C H C$ 's disclosure policy, please see the Self-Study Program.

\section{Funding}

The authors received no financial support for the research, authorship, and/or publication of this article. 


\section{References}

Binswanger, I. A., Stern, M. F., Deyo, R. A., Heagerty, P. J., Cheadle, A., Elmore, J. G., \& Koepsell, T. D. (2007). Release from prison-A high risk of death for former inmates. New England Journal of Medicine, $356,157-165$.

Brinkley-Rubinstein, L., McKenzie, M., Macmadu, A., Larney, S., Zaller, N., Dauria, E., \& Rich, J. (2018). A randomized, open label trial of methadone continuation versus forced withdrawal in a combined US prison and jail: Findings at 12 months post-release. Drug and Alcohol Dependence, 184, 57-63.

Brinkley-Rubinstein, L., Zaller, N., Martino, S., Cloud, D. H., McCauley, E., Heise, A., \& Seal, D. (2018). Criminal justice continuum for opioid users at risk of overdose. Addictive Behaviors, 86, 104-110.

Bronson, J., Stroop, J., Zimmer, S., \& Berzofsky, M. (2017). Drug use, dependence, and abuse among state prisoners and jail inmates, 2007-2009 (NCJ 250546). Washington, DC: Bureau of Justice Statistics.

Centers for Disease Control and Prevention. (2017). Opioid overdose/U.S. state prescribing rates, 2016. Retrieved from https://www.cdc.gov/drugoverdose/maps/rxstate2016.html

Centers for Disease Control and Prevention. (2018). Opioid overdose/Prescription opioid data. Retrieved from https://www.cdc.gov/drugoverdose/data/prescribing.html?CDC_AA_refVal=https\%3A\%2F\%2Fwww.cdc. gov\%2Fdrugoverdose $\% 2$ Fdata $\% 2$ Foverdose.html

Degenhardt, L., Larney, S., Kimber, J., Gisev, N., Farrell, M., Dobbins, T., ... Burns, L. (2014). The impact of opioid substitution therapy on mortality post-release from prison: Retrospective data linkage study. Addiction, 109, 1306-1317.

Marsch, L. A. (1998). The efficacy of methadone maintenance interventions in reducing illicit opiate use, HIV risk behavior and criminality: A meta-analysis. Addiction, 93, 515-532.

Mattick, R. P., Kimber, J., Breen, C., \& Davoli, M. (2008). Buprenorphine maintenance versus placebo or methadone maintenance for opioid dependence. Cochrane Database of Systematic Reviews, 16, CD002207.

National Governors Association. (2017). State reporting: NGA compact to fight opioid addiction. Retrieved from https://www.nga.org/wp-content/uploads/2019/01/NGA-Opioid-Compact-State-Reporting-Chart-003. pdf

National Institute on Drug Abuse. (2019). Opioid overdose crisis. Retrieved from https://www.drugabuse.gov/ drugs-abuse/opioids/opioid-overdose-crisis

Nellis, A. (2016). The color of justice: Racial and ethnic disparity in state prisons. Washington, DC: The Sentencing Project. Retrieved from http://www.sentencingproject.org/wp-content/uploads/2016/06/TheColor-of-Justice-Racial-and-Ethnic-Disparity-in-State-Prisons.pdf

Rich, J. D., McKenzie, M., Larney, S., Wong, J. B., Tran, L., Clarke, J., ... Zaller, N. (2015). Methadone continuation versus forced withdrawal on incarceration in a combined US prison and jail: A randomised, open-label trial. The Lancet, 386, 350-359.

U.S. Department of Health and Human Services, Office of the Surgeon General. (2016). Facing addiction in America: The Surgeon General's Report on alcohol, drugs, and health. Washington, DC: Author.

Wickersham, J. A., Azar, M. M., Cannon, C. M., Altice, F. L., \& Springer, S. A. (2015). Validation of a brief measure of opioid dependence: The Rapid Opioid Dependence Screen (RODS). Journal of Correctional Health Care, 21, 12-26. 